\title{
Characterization and eradication of the high concentration infection source of oral segment of Candida by infection examination diagnosis system
}

Yuusuke Nonomura ( $\nabla$ n-dent@tulip.ocn.ne.jp )

Nonomura Dental Clinic

\section{Research Article}

Keywords: Candida albicans, IEDS, tooth caries, infection source, infection field, diffusion field, carrier field, spread field, infection chain, relative differentiation, ventilator, receptor field, resistance field, adhesive colony, fixed colony,

Posted Date: April 22nd, 2021

DOI: https://doi.org/10.21203/rs.3.rs-150782/v3

License: (c) (i) This work is licensed under a Creative Commons Attribution 4.0 International License.

Read Full License 
Characterization and eradication of the high concentration infection source of oral segment of Candida by infection examination diagnosis system

Yuusuke Nonomura DDSc., ${ }^{* \dagger}$

$\dagger$ Addresses Nonomura Dental Clinic, National Yagata Bldg 2F 3-6, Higasiyamadouri 3-6, Chikusa-ku, Nagoya city, Aichi 4640807, Japan

* Corresponding author. E-mail: n-dent@tulip.ocn.ne.jp,

Tel: +81-52-781-4185 Fax: +81-52-781-4185

\section{Abstract}

Purpose: Candida albicans (CA) is a major pathogenic fungus that causes a critical infection and life-threatening disease. Currently, during the treatment of COVID-19 (in ICU), invasive Candidiasis and Candida colonization should be prevented to avoid the increase, in mortality, in SAPSII, and in length of stay. Our aim was to characterize and analyze the infection of CA for eradication.

Methods: We characterized and analyzed the infection source and infection field which are carrier field, diffusion field, and spread field, by using a new infection examination (diagnostic) system (IEDS) comprising a new dental formula (measurement analysis) medium (DFM) with time, space, and phase, and the simultaneous relative differential equations (C4RDE).

Results: This study showed that CA was not an endogenous microorganism, but a fungus that infected teeth causing dental caries that was successfully eradicated from all teeth of 353 Japanese patients.

Conclusion: Our methods help to eradicate the infection source, prevent critical infection, and reduce mortality. The IEDS is a powerful tool that can be applied for any infection easily. It is possible for the dentists to characterize CA by IEDS and eradicate CA from the oral cavity. IE(D)S will dramatically reduce the threat to the living body by pathogenic microorganisms.

Keyword: Candida albicans, IEDS, tooth caries, infection source, infection field, diffusion field, carrier field, spread field, infection chain, relative differentiation, ventilator, receptor field, resistance field, adhesive colony, fixed colony,

\section{Abbreviation (see Supplementary Abbreviation)}




\section{Introduction}

Candida albicans (CA) is a fungus that causes a life-threatening infection [1-3]. Furthermore, CA colonization on airway is associated with pneumonia, malignant tumors, failure of organ transplantation, ocular opacities, digestive disorders, and respiratory disorders [3-11]. Moreover, a recent study [12] suggested an adverse influence of COVID-19-associated pneumonia with CA, on the oral cavity, pharynx, heart, liver, spleen, central nervous system, urinary organs, eye, skin, vagina, and the endocrine system, any of which can be the cause of the infection $[1,2]$. This leads to a synergistic biofilm formation which causes virulence [13]. Conventionally, as CA is considered an endogenous microorganism, there are few existing methods of disinfection of CA from a living body or the prevention of transmission to living body.

\section{Materials and Methods}

In our study, we have eradicated CA from all the teeth of 353 Japanese patients and showed that CA was not an endogenous microorganism (Tables 1,2 ). The receptive property, resistance property, adhesion property (the condition equivalent to colonization [3-10], and fixing property (condition equivalent to pneumonia $[3,10]([4-9]$ may be included.) of the examination tissue and teeth are indicated by the infection examination system (IES) (Figs. 1, 2) which is the new dental formula (measurement analysis) medium (DFM) with time, space, and phase (Figs. 3-5) (Supplementary Notes S1-S27) and the simultaneous relative differential equations (C4RDE), calculated as the infection examination diagnosis system (IEDS) [14] especially the RDE (SOC). the observation object itself takes arithmetic circuit and display, one can observe phenomenon, directly [14](Fig. 1).

The medium is observed as the infection source (IS) (field), diffusion field (DF), spread field (SF), and carrier field (CF), which comprise the infection field (IF), and the continuum of the infection field (IF) is the infection chain (IC). (Figs. 1 and 2, and in Supplementary Notes S1-S10)

The fields and IS are expressed by the two equations in C4RDE [14], which describe the active property (active coefficient $\varepsilon_{\mathrm{a}}$ ) and the decline property (decline coefficient $\varepsilon_{\mathrm{d}}$ ) for the IF. In addition, the equation ( $\mathrm{C} 4 \mathrm{RDE}$ ) provides the infection solution and the defense solution from the medium. The solutions are defined by infection function (infection coefficient $\gamma_{\mathrm{i}}$ ) and defense function (defense coefficient $\gamma_{\mathrm{d}}$ ). For each of the two or more factors (the number of factors is unrestricted), the active coefficient and decline coefficient are subtracted and added. This differentiation does not include rotation and vibration. It does not have an error with an infinitesimal real number. Therefore, The RDE is the only equation whose simultaneous is possible [14].

\section{Each field and IC}

In the diffusion field (DF), microorganisms diffuse from IS by potential energy. The spread field (SF) is defined as the spread, without a concentration gradient. The carrier field (CF) is defined as the field in which microorganisms are transferred by carrier (e.g., ventilator, toothbrush, floss, and 
food). The infection field (IF) consists of the IS (in the segment) and the three fields (DF, SF, and CF). The continuum of the IF is the IC. In the IF, microorganisms encounter an organ. If the microorganism can adhere (adhesion stage colonization) to the substance that encountered the receptor field $(\mathrm{RcF})$, it will colonize the substance. In suitable conditions, the proportions of microorganisms increase (fixing stage colonization), the IS will be generated, and the IF will progress. The IF begins from the IS and is infected in three ways (IFF is infection field function): DF, SF, and CF. In the IS, contaminated substances are generated by the IF.

The IEDS aims to stop the IC by eradication of IS, disinfection of CF, investigation of resistance field (RsF), and obstruction of RcF-ized for the substance.

\section{Medium (DFM)}

A sterilization swab (SS; trace) of the whole tooth without contact with the adjacent tooth (Fig. 3a) was performed. Then, the swab was applied to the area of dental formula (The trade mark and pat of dental formula, MicroDent Co., Ltd.) on corresponding medium (The CHROMagar ${ }^{\mathrm{TM}}$ Cnadida, BD BBL $^{\mathrm{TM}}$, Becton, Dickinson and Company,) (Supplementary Notes S11-S13).

Medium (DFM): CHROMagar was used. Most of the fungi detected were Candida albicans. It can be sampled and cultivated at $37{ }^{\circ} \mathrm{C}$ for $48 \mathrm{~h}$

The colony concentration (CC) on the medium in this sampling was calculated by the following equations. CAC refers to Candida albicans concentration.

CC of each dental formula area on the medium

$\propto$ CAC of the tooth part (teeth parts) in contact with the SS (CWSS)

+ CAC in the saliva adhering to the teeth in CWSS

+ CAC in the saliva that is in the environment of CWSS

+ CAC of the (marginal) periodontal tissue in CWSS

+ CAC of the air in CWSS

This sampling includes swabs of the tooth, periodontal tissue, saliva, and air, and is the sum of the four parts. The CAC of tooth in contact with the SS includes CA and the IF from other teeth and $\operatorname{organ}(\mathrm{s})$.

1. The all-teeth examination (ATE) included the swab of all teeth (Fig. 3b).

2. The partial teeth examination (PTE) (tooth unit) included the swab of each tooth. The swab was applied to each area of the DFM (Fig. 3c). If a swab was negative after treatment and disinfection, this confirmed that the IS was tooth caries (Fig 4, 5).

Treatment procedure is provided in Supplement Fig S14.

C4RDE diagnosis equation is shown in Fig. 1 [14] (Supplementary SDE in C4RDE)

\section{Results}


According to C4RDE [14] and IFF (Fig. 1) corresponding to the model in Fig. 2, the infection is measured and analyzed, and it can be eliminated by treatment. The adhered (about $\gamma_{\mathrm{i}}=0$ ) and fixed colonies $\left(\gamma_{\mathrm{i}}>0\right)$ can be detected, and disinfection can occur.

Especially, CA examination diagnostics (IEDS) by space, time, and phase analyses of teeth using DFM indicated that the biggest IF (including IS) of this fungus was tooth caries. (Fig. 4; Tables 3-10, Supplementary Table S23: space analysis) (Supplementary Notes S1-S28 and Supplementary Figs. S2-S5: time and phase analysis) (Supplementary Tables S1-S22). It is thought that the characteristics of IF and IC acquired from space, time, and phase analyses are useful for disinfection, prevention of transmission, and the control of microorganisms; however, they have also suggested the analysis of other microorganisms and viruses.

Treatment based on IEDS revealed that the CA elimination rate in the oral segment is approximately $100 \%$ as a result of elimination of dental caries and cleaning (Figs. 4, 5, Table 6, and Supplementary Fig S14). The combined treatment of dental caries and tooth cleaning by the antifungal agent [15] resulted in the eradication of CA by approximately $100 \%$ in the oral segment of the part of the IC.

CA and DMFT index (WHO) is decayed, missing, and filled tooth index.

Incidentally, in the first examination, the results were: CA (+) 40.5\% (DMFT: 62.8\%), CA (-) 56.4\% (DMFT: $47.1 \%$ ). The significant difference of DMFT was $15.7 \%(\mathrm{P}<0.01)$. This showed that $\mathrm{CA}$ is not an endogenous microorganism. It suggests a relationship with dental caries (Table 1 and Supplementary Tables S1-S8).

\section{CA in a dental-caries tooth}

The IS is defined as the space that is colonized (adhesion stage or fixing stage) by microorganism (in the shape of microscopic image of caries tooth, multi formation images, such as fungi mycelium and spore, are seen (Supplementary Fig. 1)).

\section{Fields}

1. The main RcF is dental caries.

2. DF is about one tooth.

3. SF is not observed in CA; LB is observed.

4. $\mathrm{CF}$ is evaluated by the sampling by a swab; therefore, toothbrushes, ventilator, fine droplet nuclei, respiratory drop, ejected contaminated water drops by dental air turbine, and food are considered to be important CFs.

5. The tooth has a powerful RsF.

6. IF is eliminated in the oral segment.

7. IF is mathematically equivalent to any segment. It can be calculated for complicated systems using only two kinds of coefficient (active coefficient $\varepsilon_{\mathrm{a}}$ and decline coefficient $\varepsilon_{\mathrm{d}}$ ).

8. The IS is detectable; therefore, it can be completely eradicated. 
9. $\mathrm{RcF}$ is detectable, and hence, investigation of the IS is easy. Moreover, RcF, which is the risk field of infection, can be eliminated, and infection can be prevented.

10. As RsF is detectable, the prevention of transmission is possible.

Space analysis by dental formula (Supplementary Notes S18-S24)

The local IS indicated that teeth caries included the fixing colonization part of the CA (Fig. 4, Tables 3-10, and Supplementary Table S21). CA was detected in $243(68.3 \%)$ teeth caries (C1 to C3 in 356 teeth). In adjacent healthy teeth (true healthy tooth [Adj-THT] + restored healthy teeth [Adj-RHT]) and non-adjacent healthy teeth \{true healthy tooth [nAdj-THT] + restored healthy teeth [nAdj-RHT]), the values were 46 (12.9\%) and 57 (16.0\%), respectively.

When CA was present in many teeth, in the oral segment, it appeared to be an endogenous microorganism (Fig. 3e and 5) [22]. In contrast, when CA infections was present in a few teeth, it was fixed to the tooth and caused teeth caries (Fig. 3d and 4). A CA(+) caries tooth and a CA(-) caries tooth existed in the same mouth. (Supplementary Table S18). These indicate existence of a RsF, because, infection cannot be realized with the influence of CF because of tooth brushing.

\section{Phase analysis by dental formula}

1. The phase of time is observed as Fig4 and 5. It is useful for the prediction of infection.

2 The phase of space is observed as Fig4 and 5. It finds one IS buried in multiplex IS.

3. The phase of microorganisms (Especially, Only phase analysis, It define as comparison of multiple microorganisms.) It detects RcF or RsF. An interesting relationship between RsF and Lactobacillus (LB) is indicated (Supplementary Fig. 5).

\section{Re-infection}

Moreover, light adhesion (adhesive colony) was observed in some cases which could lead to re-infection; however, these cases were easily removed.

\section{Discussion}

There are many examples that omit the IEDS based on specific space, time, and phase of the IF, and these are confusing. There are studies describing the involvement of dental caries [15-18]; in contrast, there are studies not related to dental caries $[19,20]$. It may be the feature of CA that discussion in [3-12] similar to VAP has generated. In these studies, there was no analysis of the infection caused by $\mathrm{CA}$, the application of the antifungal agent was stopped, and there was no plan against re-infection; moreover, studies have been reported regarding the use of antifungal agents for infection in root canal (Pseudomonas [9] is treated as an important pathogenic bacteria of apical periodontitis), but there is a lack of studies on the propriety of application of the antifungal agent after disinfection, and the technique and extent of maintenance to achieve permanent prevention of transmission and full disinfection. Therefore, these methods have not resulted in the eradication of infection. 
In the space analysis of the $\mathrm{CA}(+)$ non caries tooth we found that in 32 of 46 adjacent healthy teeth with $\mathrm{CA}(+)$, CA was removed by professional cleaning or brushing (after dental caries operation); (Table 5a). In 36 of 57 non-adjacent healthy teeth with $\mathrm{CA}(+)$, CA was removed by professional cleaning or brushing; (after dental caries operation) (Table 5b).

These are considered to be light CA infections, which depends on the internal transmission by IF (from the caries teeth), or on the external transmission (food, dust splash, hospital infection, etc.) by IF, and adhesion. Internal transmission was considered for DF that was less than one tooth away (Fig. 2, Supplementary Figs. 11-13).

\section{Four-level classification}

In our study, the infection of CA was classified into one of the following four levels:

1. Encountering tissue (There is no invasion)

2. Adhesive colonization (surface colonization) (Supplementary Note S14) (colony equivalent to colonization [3-10]). This level is removable by cleaning.

3. Fixing colonization (invasive colonization) (Supplementary Note S15) (In the lung, the fixing colony will cause pneumonia.) [3, 11], It causes a infection.

4. Outbreak (of colonization) in organs (tissue, substance) (Supplementary Note S16)

The first-and second-level CA (from all true healthy teeth and the restored healthy teeth in Table 7) can be mostly disinfected by cleaning; however, at the third level, disinfection cannot be accomplished without treatment. The fourth level is a colony concentration (CC) of 100 or more, and a specific exponential increase was also observed on the chart (Table 7).

5. Four colony level (see Supplementary Detail (contents) of colony level)

Colony level 1: adhesion level (1-4) (The number of colony)

Colony level 2: transient level, increased growth (5-14)

Colony level 3: fixing level, increased growth (15-99)

Colony level 4: outbreak (100-)

\section{Adhesion}

The result of CA adhesion in healthy tooth is given in (Table 5). CA was detected on teeth, except for the caries tooth. It was not a fixed CA, but an adherent CA. We assumed the possibility of internal transmission from the $243 \mathrm{CA}(+)$ caries tooth to the others. We found that in 32 teeth, adhesive colonization was removed by tooth cleaning. Among the non-adjacent healthy teeth, in which there was a strong possibility of internal transmission and/or external transmission, 36 CA (+) teeth had removed adhesive colonization by tooth cleaning, which accounted to a total of 311 teeth $(87.4 \%$; 311/356). Furthermore, the dummy is the result of the external and/or internal transmission; therefore, 10 teeth can be added to the total which resulted in $90.2 \%(321 / 356)$ of the teeth. If the 35 unclear teeth are removed, this becomes $100 \%$ (321/321). Therefore, it is thought that caries tooth occurs from a fixed CA. The IS is a caries tooth, which was treated 
with an antifungal agent, resulting in disinfection (Supplementary Note S24). If CC in each dental formula area is 0 , it must also be 0 in saliva, in periodontal tissue, and in the air.

\section{The oral segment is the entrance for the living body.}

For the oral segment, which is the entrance for the living body, to become CA-free, the infection chain (IC) must be broken in the connected segment that could be infected from the oral segment. There is a strong possibility that the teeth constitute the first habitat of CA; thus, CA disinfection of the teeth was very effective (Supplementary Notes S26-S27). Moreover, resin filling, artificial tooth for the final prosthetics, denture and healthy teeth, which are clear on DFM, also occasionally have adherent CA. The information from IEDS can examine the IR (IR subtracts IS from IC), but in many cases, it is possible to maintain a patient's oral segment CA-free continuously. In the oral segment, which is the entrance to the living body, ref. 2 (P87) suggests food as the source of IF (Japan is an exception [23]), IES shows the specific dental caries are because of infection from a source other than food. The treatment, based on detailed measurement analysis of the IF, shows that the IC can be eliminated, including internal and external infections from the natural environment.

\section{No endogenous microorganism}

In the oral segment, one or more CA-infected teeth may exist. In the case of the latter, two or more $\mathrm{IF}(\mathrm{s})$ (and accordingly, DF, SF, and CF) exists in the restricted space of the oral segment; thus, the $\mathrm{IF}(\mathrm{s})$ suggest that $\mathrm{CA}$ is the causative endogenous microorganism. Importantly, there is a possibility that RsF is powerful in the oral segment. Moreover, it is thought that the reason for the negative value of ATE was the small amount of external transmission.

This may indicate that the prevalence of CA(s) in Japan, such as in food, soil, and water, was very low (Table 1). Furthermore, the Food Sanitation Law of Japan [23] does not recognize the contamination of pathogenic microorganisms. The same is true of the Water Supply Law [24].

\section{The characterizations of $\mathrm{CA}$}

CA must consider the phase of the space-time of infection source, infection field, receptor field, resistance field, adhesive colony, fixed colony and infection chain. Moreover, CA must consider the phase of microorganisms. If they are neglected, in the statistical research or the medical treatment etc., the study cannot obtain the correct result.

\section{Conclusion}

In the treatment and prevention of infection, specification of the characteristics of IS and IF of the microorganisms is the highest priority. The IEDS, including the DFM, indicates the exact DF, SF, CF, RsF, RcF, dental caries time, IC, and other properties of microorganisms such as CA, and can simultaneously show the infection route and infection medium (Fig. 1, 2) (Supplementary Note S28). The surveillance of CA disinfection and the information from IEDS of specific teeth are very effective in the prevention of infection by CA. 
It is necessary to perform dental formula examination of the microorganisms contained in dental caries and group it according to three phases (the infrared analysis method is important for Streptococcus mutans). Accordingly, information from IEDS can prevent infection, while preventing various diseases in other internal organs. Additionally, it is able to perform high-precision dental caries treatment (including operation), and prevent tooth loss. It is necessary to classify and verify the internal transmission from the untreated fixing teeth and active internal transmission by dental operation.

The outer CA infection routes (IR) include soil, food, pets, and care, through which progression can occur. Inner infections can transfer from the oral segment to respiratory organs or digestive organs, nasal cavity, and paranasal sinus. Moreover, disinfection of the ventilator used for treatment is important.

As mentioned above, the field of CA is mainly observed as CF.

\section{The use of the ventilator}

Although the use of the ventilator through the oral cavity in which dental-caries tooth exists is not recommended, it is inevitable in cases of infection. Therefore, in cases of infection, medical treatment is recommended. IEDS is very effective in prevention of hospital infection.

\section{Solutions}

${ }_{1} \mathrm{~N}$, is the other microorganisms and antibacterial factors, ${ }_{2} \mathrm{~N}$ is the number of CA. In the treatment, the active factor of CA is only the growth factor (due to the influence of RcF and RsF ). The $\gamma_{i}=0.4-$ 0.5[25]. Correspondingly, the control of $\gamma_{\mathrm{i}}$ and/or $\gamma_{\mathrm{d}}$ is important for treatment and prevention. The doctor should be treated as $\gamma_{\mathrm{d}}>0$. (Supplementary POE S1)

It is important that doctors increase the number ${ }_{1} \mathrm{~N}$ of inhibitory factors. In addition, IE(D)S shows the interaction of two or more kinds of microorganisms, such as CA and LB.

\section{Summary}

Eradication of the infection source (IS) of the oral segment(s) and breaking the infection chain (IC) by IE(D)S will dramatically reduce the threat to the living body by pathogenic microorganisms. 


\section{Declarations}

\section{Funding}

'Not applicable'

\section{Conflicts of Interest}

The author owns the patents, trademark, and copy right of DFM.

1) 2003-381874, Dental diagnostic system, November 12, 2003, in Japan

2) 2003-421304, Dental formula system, December 18, 2003, in Japan,

3) Japanese Pat No.5337353, 2007-138463, Dental formula Apparatus, May 24, 2007, in Japan

4) 2008-209406, Infection inflammation immune response measuring diagnostic apparatus, August 18, 2008, in Japan,

5) 2020-41422 Arithmetic unit, March 10, 2020, in Japan, especially regarding details of C4RDE,

6) Japanese Pat No6555573, 2019.8.7, Computer arithmetic unit, Riken Co., Ltd.,

7) Japanese Pat No6555574, 2019.8.7, Computer arithmetic unit, Riken Co., Ltd.,

8) Japanese Pat No6796295, 2020.11.18, Str Arithmetic unit, Riken Co., Ltd.,

9) Japanese Pat No6800460, 2020.11.27, Arithmetic unit, Riken Co., Ltd.,

10) Trade Mark of Dental Formula (Measurement Analysis) Medium (DFM), MicroDent Co., Ltd., No5341950

11) Copyright of dental formula (measurement analysis) medium (DFM), MicroDent Co., Ltd.,

\section{Ethics approval}

See Supp. Figure 0 no ethics problems,

\section{Consent to participate}

The clinic has always disclosed that treatment data without personal data will be used for research (See Supp. Figure 0), and no participant (patients) has ever objected to this.

\section{Consent for publication}

The clinic has always disclosed that treatment data will be used for research (See Supp. Figure 0), and no participant has ever objected to this.

\section{Availability of data and material}

See Supplementary information,

\section{Code availability}

'Not applicable' 
Figure Legends

\section{Infection Chain}

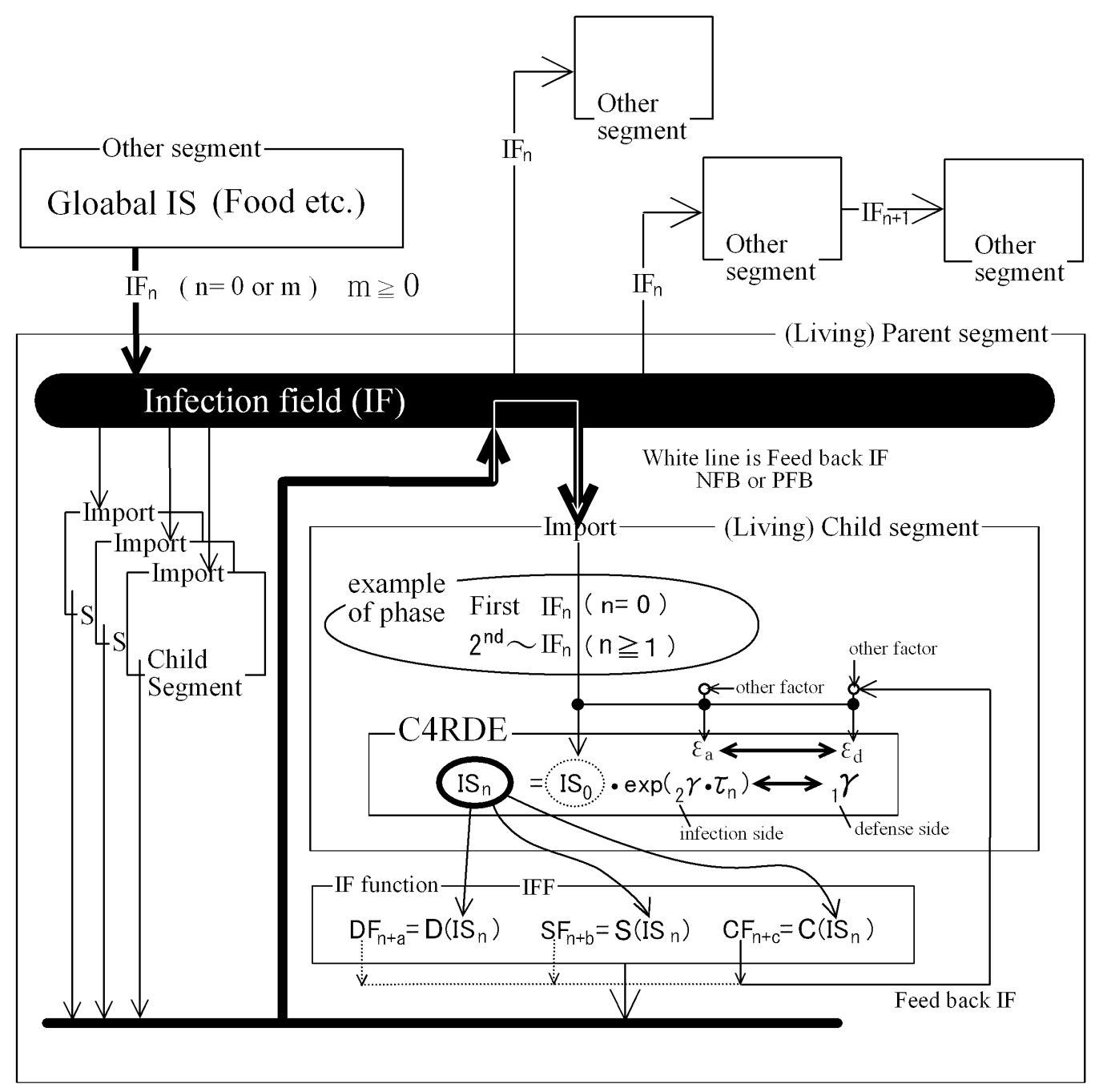

IF : Infection field

DF : Diffusion field

SF : Spread field

CF : Carrier field

IS : Infection source
RcF : Receptor field

RsF : Resistant field

SOI : Solution of infedction SOD : Solution of defens

$\varepsilon_{\mathrm{a}}$ active coefficient $\mathrm{m}>0$ : integer

$\mathrm{n}>0$ : integer

a, b, c : integer

$\varepsilon_{\mathrm{d}}$ decline coefficient

Fig. 1 Definition of infection chain (IC) and various segment(s) 
The infection source (IS) is defined as the space colonized by microorganisms. In the diffusion field (DF), microorganisms diffuse from the IS by potential energy. The spread field (SF) is defined as the spread that is not dependent on the concentration gradient. The carrier field (CF) is a field in which microorganisms are transferred by a carrier (e.g., toothbrush, floss, food). The infection field (IF) consists of the IS (in the segment) and three fields (DF, SF, and CF). The continuum of the IF is the IC (Fig. 3 and Supplementary Note S1-S10). They were measured in dental formula medium by C4RDE (C4SDE), which analyzes IF (including IS).

$\varepsilon_{\mathrm{d}}$ : Inhibition term (treatment, prevention, medicine, defense, decline term), as the relative decrease in factor and antigen: Decline coefficient $\varepsilon_{\mathrm{d}}$

$$
\begin{gathered}
\varepsilon_{d}=\varepsilon_{\mathrm{d}_{-} \mathrm{RsF}}+\varepsilon_{a_{-} \overline{\mathrm{DF}}}+\varepsilon_{a_{-} \overline{\mathrm{SF}}}+\varepsilon_{a_{-} \overline{\mathrm{CF}}}+\varepsilon_{\mathrm{d}_{-} \mathrm{trt}} \\
\varepsilon_{d}=\varepsilon_{\mathrm{d}_{-} \mathrm{RsF}}+\varepsilon_{\mathrm{d}_{-} \text {trt }} \text { (without anti field.) }
\end{gathered}
$$

$\varepsilon_{\mathrm{a}}$ : Infection term (microorganism term, CA term) as the relative increase in factor and antigen: Active coefficient $\varepsilon_{\mathrm{a}}$

$$
\varepsilon_{a}=\varepsilon_{a_{-} \mathrm{RcF}}+\varepsilon_{a_{-} \mathrm{DF}}+\varepsilon_{a_{-} \mathrm{SF}}+\varepsilon_{a_{-} \mathrm{CF}}+\varepsilon_{a_{-} \mathrm{growth}}
$$

C 4 RDE with $\varepsilon_{\mathrm{d}}$ and $\varepsilon_{\mathrm{a}}$

$$
\begin{aligned}
& \mathrm{K}
\end{aligned}
$$

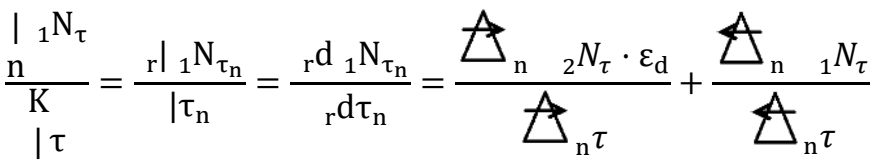

$$
\begin{aligned}
& \text { n } \\
& \text { K }
\end{aligned}
$$

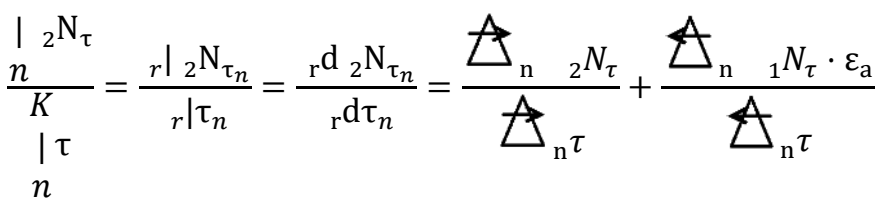

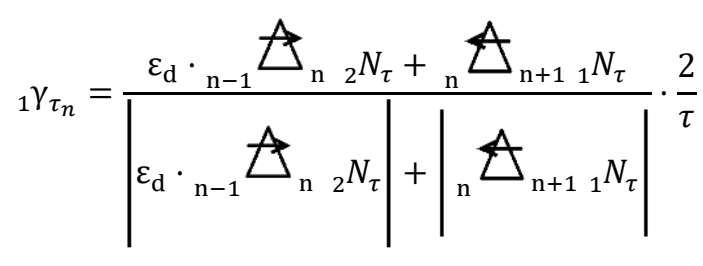$$
{ }_{2} \gamma_{\tau_{n}}=\frac{\mathbb{\mathrm { n }}-1_{\mathrm{n}{ }_{2} N_{\tau}+\varepsilon_{\mathrm{a}} \cdot{ }_{\mathrm{n}}} \mathbb{\Delta}_{\mathrm{n}+1{ }_{1} N_{\tau}}}{\left|\mathrm{n}_{\mathrm{n}{ }_{2} N_{\tau}}\right|+\left|\varepsilon_{\mathrm{a}} \cdot{ }_{\mathrm{n}} \mathbb{\Delta}_{\mathrm{n}+1{ }_{1} N_{\tau}}\right|} \cdot \frac{2}{\tau}
$$

$$
\begin{aligned}
& { }_{1} \mathrm{~N}_{\tau_{\mathrm{n}}}={ }_{1} \mathrm{~N}_{0} \cdot \exp \left({ }_{1} \gamma_{\tau_{\mathrm{n}}} \cdot \tau_{\mathrm{n}}\right) \\
& { }_{2} \mathrm{~N}_{\tau_{\mathrm{n}}}={ }_{2} \mathrm{~N}_{0} \cdot \exp \left({ }_{2} \gamma_{\tau_{\mathrm{n}}} \cdot \tau_{\mathrm{n}}\right)
\end{aligned}
$$




$$
{ }_{1} \mathrm{~N}_{\tau_{\mathrm{n}}}={ }_{1} \mathrm{~N}_{0} \cdot \exp \left({ }_{1} \gamma_{\tau_{\mathrm{n}}} \cdot \tau_{\mathrm{n}} / \tau_{\mathrm{r}}\right)
$$

${ }_{1} \mathrm{~N}_{\tau \mathrm{n}}$ is the value of the inhibitor, and ${ }_{2} \mathrm{~N}_{\tau \mathrm{n}}$ is the value of the pathogenic object (in the case of CA) (Supplementary POE S1). The $\tau$ ratio $\left(\tau_{\mathrm{r}}\right)$ is used if the reaction time of existence 1 ( $\left.{ }_{1} \mathrm{~N}\right)$ and existence $2\left({ }_{2} \mathrm{~N}\right)$ is different. ${ }_{1} \mathrm{~N}_{0}$ is the initial value of the inhibitor, and ${ }_{2} \mathrm{~N}_{0}$ is the initial value of the pathogenic object (in the case of CA). The $\tau$ ratio $\left(\tau_{r}\right)$ is used if the reaction time of existence 1 ( ${ }_{1} N$ ) and existence $2\left({ }_{2} \mathrm{~N}\right)$ is different [14]

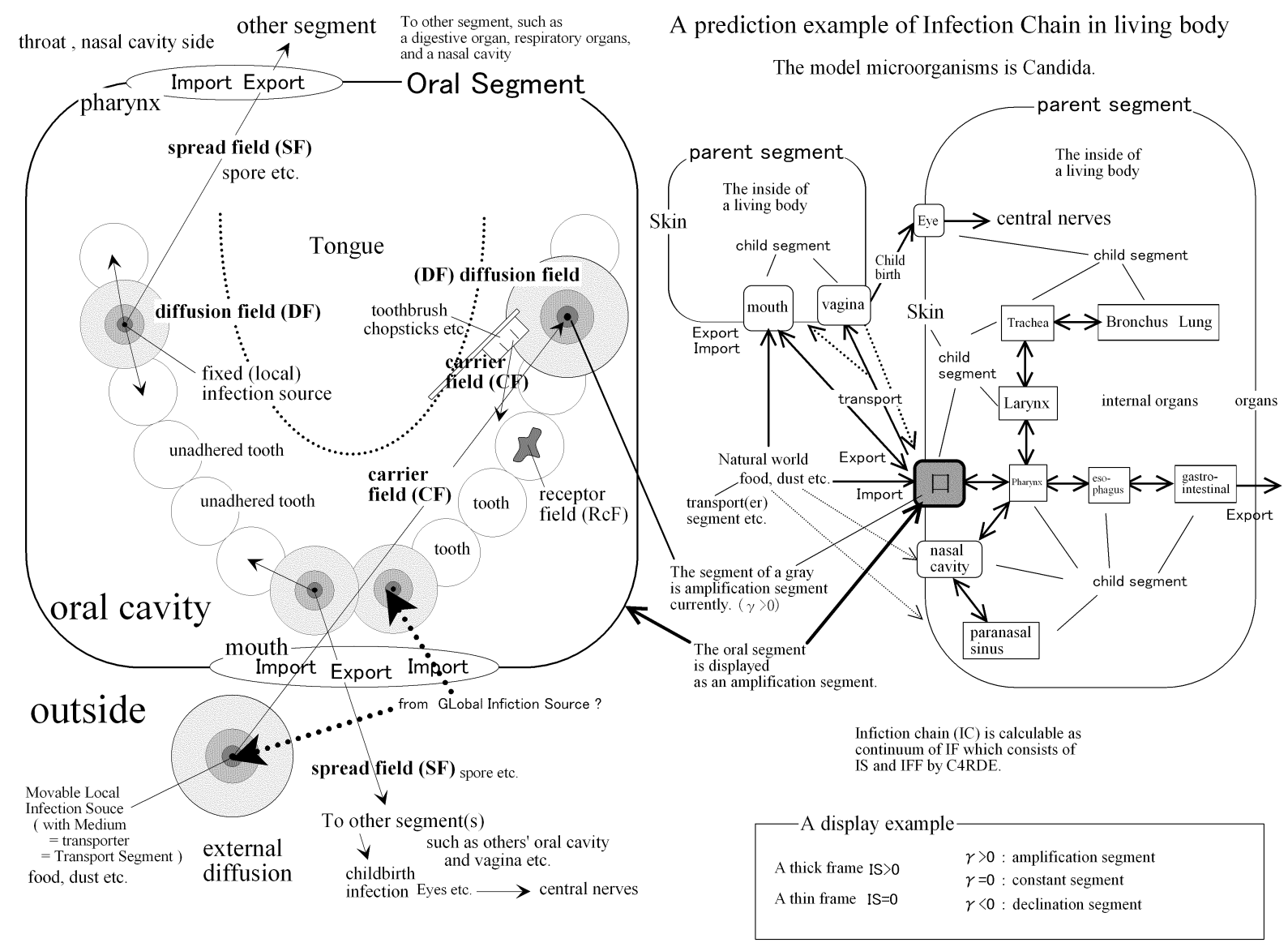

Fig. 2 Each field in the oral segment and an example of the infection chain in living body. (In the case of Candida).

The amplification segment is an example of an oral segment. Thus, the IS and infection coefficient $\gamma$ is displayed, showing cooperation with other regions. The transmission of infection can occur by the oral segment to the lung (including the airway), oral segment to the vagina at the time of childbirth.

It is thought that this relatively easy examination, analysis, and diagnostics are the most fundamental and important examinations in the prevention of infection by CA in the living body, including for dental caries tooth treatment 


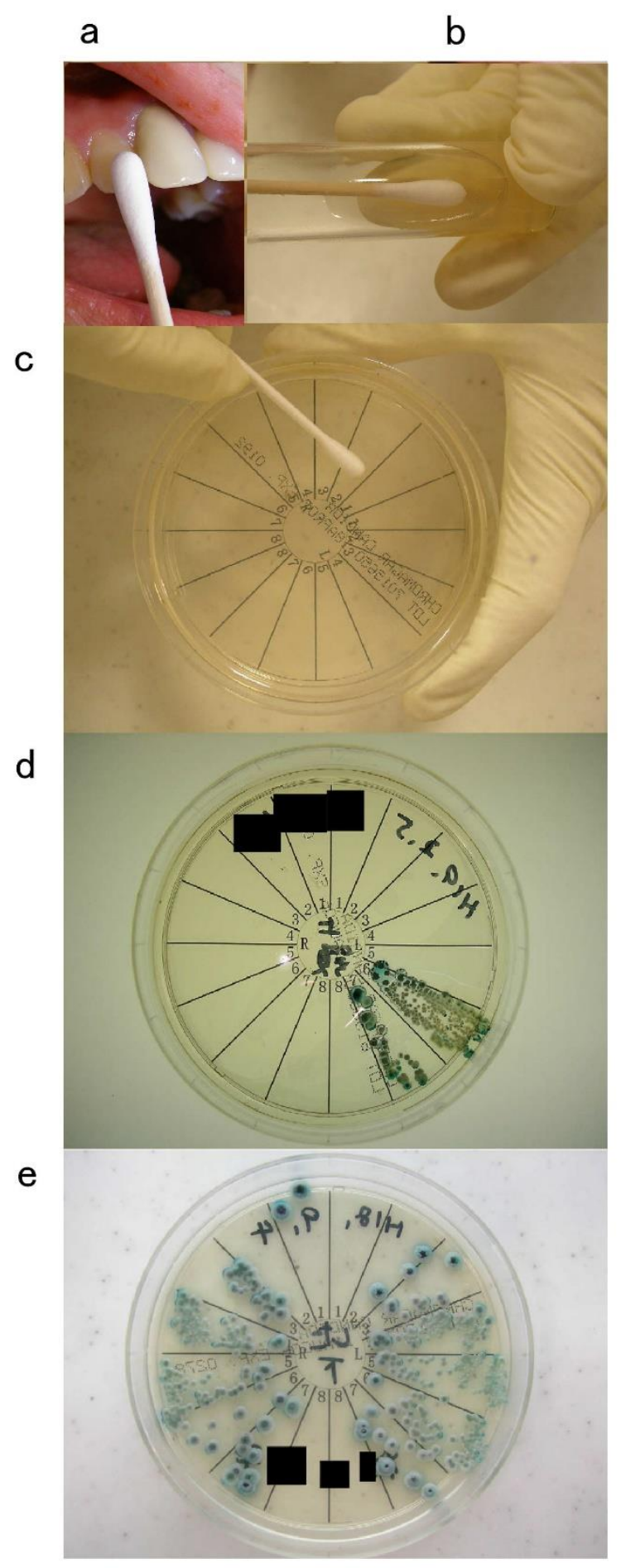

Fig. 3 The sampling method of a sample by dental formula medium (DFM),

a, A sterile swab of the teeth (crown). (Additionally, tracing the teeth cervix traces the entrance of 
the periodontal pocket, and samples soft tissues, such as the marginal gingiva and interdental papilla.) b, All teeth swabs were applied to medium (oral cavity unit examination). The all teeth examination (ATE), c, The sterile swab applied to the tooth that corresponded to each dental formula and the swab was applied to the area of dental formula on corresponding medium. The partial teeth examination (PTE) (tooth unit), d, As one or more CA-positive caries were observed, the infection source (IS) was tooth decay. e, As many CA-positive caries were observed, growth similar to that of endogenous microorganisms occurred. c-e, Dental formula medium (DFM) analyzes the sample according to the dental formula. The dental formula information on this DFM permitted space, time, and phase analyses (Table 3). The results in DFM clarified the infection by CA (Fig. 3d and e).

1. Space analysis shows the above DF, SF, CF, and the character of RcF $\{\mathrm{CA}(+)$ dental caries $\}$ where it adheres specifically.

2. Time analysis shows the infection tendency in real-time and the growth trends in dental caries over time.

3. Phase analysis shows the phase difference of Lactobacillus (LB), SF, and RsF (Supplementary Fig. 5). 

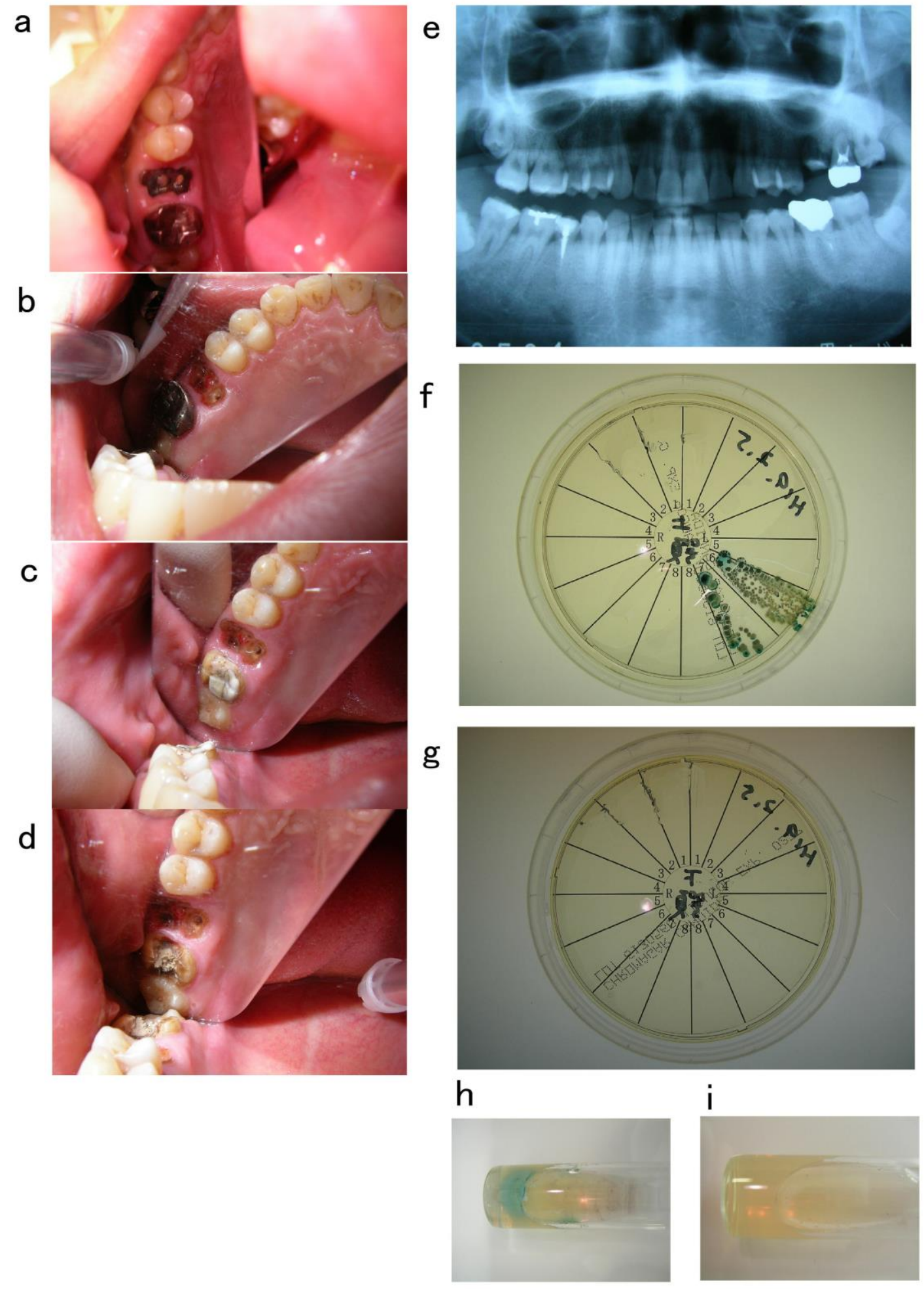

Fig. 4 Disinfection case, a few positivity tooth, 
a, Before the operation, the upper left teeth No. 6 and 7 show dental-caries. X-ray findings showed caries in No 6 and 7. b, The caries in the upper left No. 6 tooth were removed. c, The upper left No. 7 crown was removed. d, The upper left No. 7 core was removed. e, Orthopantomography at the first dental examination. f, Dental formula medium upper jaw is CA colony level 4 (100- colony counts, the level of outbreak ) in No. 6 tooth and level 3 (15-99 colony counts, fixing level with increased growth) in No. 7 tooth. g, Dental-formula medium lower jaw is clear. h, Before treatment, all teeth were CA-positive. i, After treatment, all teeth were CA-negative 

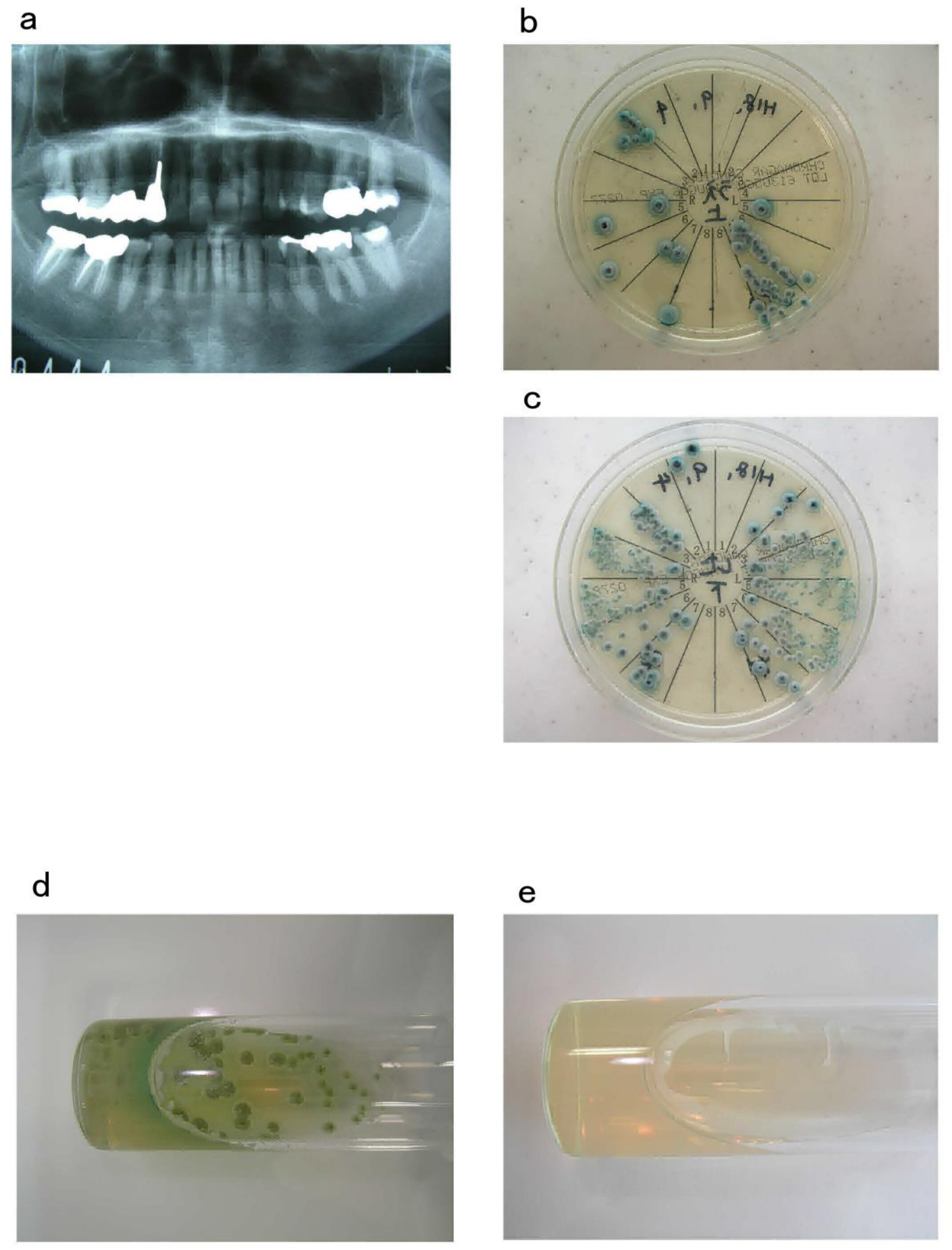
Fig. 5 Disinfection case, many positive teeth,

a, Orthopantomography at the first dental examination, b, Dental-formula medium upper jaw c, Dental-formula medium lower jaw. In this case, many teeth were CA infected. CA in the oral segment appears to be an endogenous microorganism. d, Before treatment, all teeth were CA-positive. e, After treatment, all teeth were CA-negative 


\section{Tables:}

Table 1: First examination of 353 patients (using CHROMagar medium)

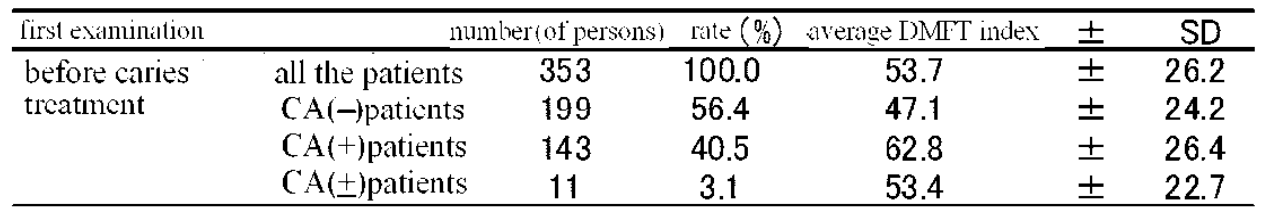

Table 2: Candida albicans (CA) examination and DMFT in the whole (teeth, periodontal tissue) oral cavity

\begin{tabular}{llccccc}
\hline lirst examination & \multicolumn{2}{c}{ numbertof persons) } & rate $(\%)$ & average DMFT index & \pm & SD \\
\hline beforc caries & CA(-)patients & 199 & & 47.1 & \pm & 24.2 \\
treatment & CA(+)patients & 143 & 100.0 & 62.8 & \pm & 26.4 \\
& CA(+1)patients & 45 & 31.5 & 55.5 & \pm & 22.6 \\
& CA(+2)patients & 43 & 30.1 & 67.0 & \pm & 24.0 \\
& CA(+3)patients & 50 & 35.0 & 63.0 & \pm & 30.0 \\
& CA(+4)patients & 5 & 3.5 & 91.2 & \pm & 15.9 \\
\hline
\end{tabular}

Table 3 Space analysis operator
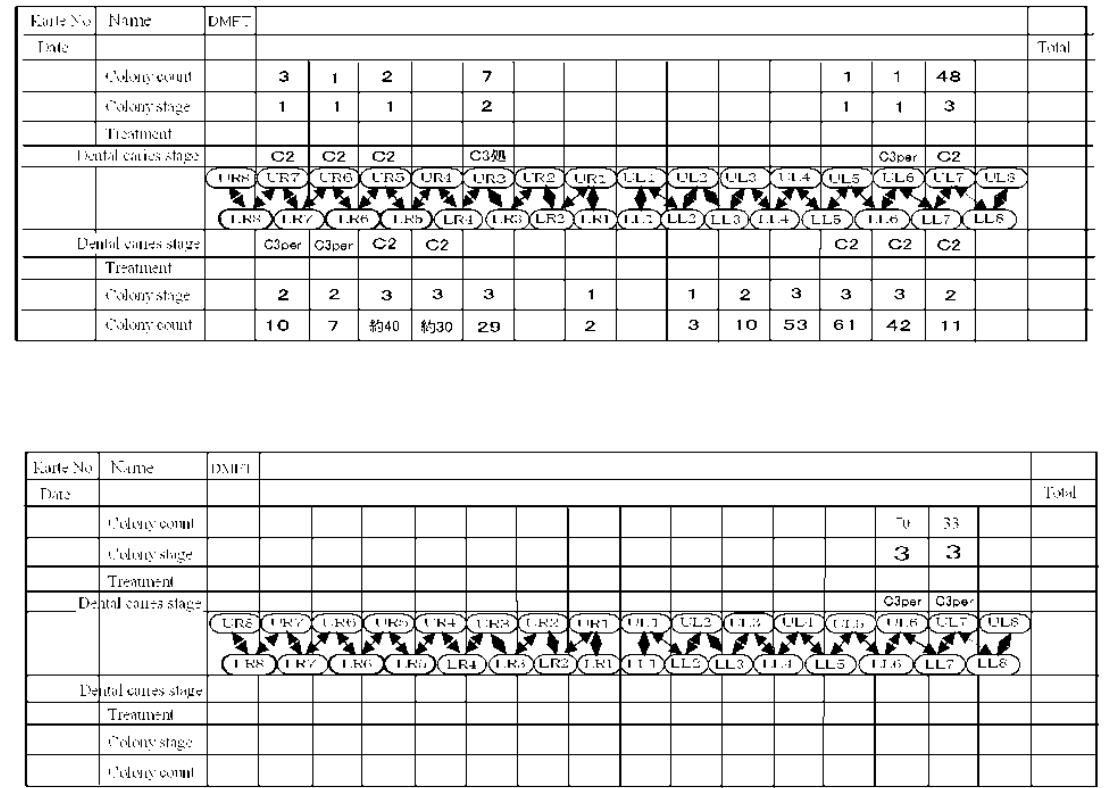
Table 4. CA (CHROMagar) examination of 49 patients, 19.8 remaining teeth

\begin{tabular}{|c|c|c|c|}
\hline 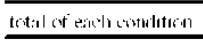 & The mumber of CA colony detection tooth & 356 & 100.0 \\
\hline & G1 & 2 & 0.6 \\
\hline & $\mathrm{C} 2$ & 124 & 34.8 \\
\hline & C3pul & 10 & 2.8 \\
\hline $\operatorname{colls}(\sin \pi)$ & C3per & 65 & 18.3 \\
\hline 243 & C3 restored tooth & 42 & 11.8 \\
\hline & adjacent true healthy tooth & 27 & 7.6 \\
\hline 46 & adjacent restored healthy tooth & 19 & 5.3 \\
\hline 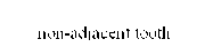 & non-adjacent true healthy tooth & 23 & 6.5 \\
\hline 57 & non-adjacent restored healthy tooth & 34 & 9.6 \\
\hline 10 & Dumy $\left(P_{0}\right)$ & 10 & 2.8 \\
\hline
\end{tabular}

Table 5 Whole circumstances of colony detection for teeth, except for teeth with caries in Table 4

Table 5 a

\begin{tabular}{cc}
\hline contents & Number (tooth) \\
\hline negative & 32 \\
attending the hospital is impossible & 10 \\
During dental treatment & 0 \\
During professional cleaning & 4 \\
Tooth extraction required & 0 \\
Total of result of the adjacent tooth with the CA colony & 46 \\
\hline
\end{tabular}

Table $5 b$

\begin{tabular}{cc}
\hline contents & Number (tooth) \\
\hline negative & 36 \\
attending the hospital is impossible & 16 \\
During dental treatment & 1 \\
During professional cleaning & 4 \\
Tooth extraction required & 0 \\
Total of result of the non-adjacent tooth $w$ ith the CA colons & 57 \\
\hline
\end{tabular}

Table 6 Disinfection results

\begin{tabular}{lcc}
\hline & Number of people & Rate \\
\hline Before dental treatment CA(+) & 143 & $\mathbf{1 0 0 . 0}$ \\
Dental treatment impossible patient & 7 & 4.9 \\
The progress unknown during dental treatment etc. & 67 & 46.9 \\
1st treatment, the end of dental treatment & 69 & 48.3 \\
After the end of 1st dental treatment. CA(-) & 68 & \\
After the end of Istdental treatment. CA(+) & 1 & 0.7 \\
After the end of Ist dental treatment. Decreasing & 1 & \\
After the end of 1st dental treatment. disinfection & 68 & 98.6 \\
2ud treatment, the end of dental treatment & 1 & \\
After the end of 2nd dental treatment. CA(-) & 1 & 100.0 \\
After the end of 2nd dental treatment, CA(+) & 0 & 0.0 \\
After the end of 2nd dental treatment. disinfection & & 100.0 \\
\hline
\end{tabular}


Table 7 Stage of dental caries and colony count using CHROMagar.

\begin{tabular}{|c|c|c|c|c|c|c|c|c|c|c|}
\hline Number & C1 & $\mathrm{C} 2$ & C3pul & C3per & G3restured & n: \dj-THT & $\mathbf{n}: \mathrm{dd}_{\mathbf{j}}-\mathrm{KH} \mathrm{I}^{\circ}$ & Idj-IH' & Adj-RHT & Dumy $(\mathrm{Po})$ \\
\hline $1-4$ & 2 & 65 & 7 & 19 & 24 & 18 & 24 & 21 & 13 & 6 \\
\hline $5-9$ & 0 & 14 & 0 & 12 & 4 & 0 & 5 & 1 & 3 & 1 \\
\hline $10-14$ & 0 & 14 & 0 & 3 & 2 & 4 & 1 & 0 & 0 & 0 \\
\hline $15-19$ & 0 & 5 & 0 & 2 & 2 & 0 & 1 & 1 & 2 & 0 \\
\hline $20-24$ & 0 & 4 & 0 & 1 & 2 & 1 & 0 & 0 & 0 & 0 \\
\hline $25-29$ & 0 & 1 & 1 & 3 & 4 & 0 & 0 & 2 & 0 & 0 \\
\hline $30-34$ & 0 & 4 & 1 & 4 & 0 & 0 & 1 & 0 & 0 & 1 \\
\hline $35-39$ & 0 & 1 & 1 & 2 & 0 & 0 & 0 & 1 & 0 & 0 \\
\hline $40-44$ & 0 & 4 & 0 & 2 & 0 & 0 & 1 & 0 & 0 & 0 \\
\hline $45-49$ & 0 & 1 & 0 & 3 & 0 & 0 & 0 & 0 & 1 & 0 \\
\hline $50-54$ & 0 & 1 & 0 & 1 & 2 & 0 & 1 & 1 & 0 & 1 \\
\hline $55-59$ & 0 & 1 & 0 & 0 & 0 & 0 & 0 & 0 & 0 & 0 \\
\hline $60-64$ & 0 & 2 & 0 & 1 & 2 & 0 & 0 & 0 & 0 & 1 \\
\hline $65-69$ & 0 & 0 & 0 & 2 & 0 & 0 & 0 & 0 & 0 & 0 \\
\hline $70-74$ & 0 & 2 & 0 & 1 & 0 & 0 & 0 & 0 & 0 & 0 \\
\hline $75-79$ & 0 & 0 & 0 & 1 & 0 & 0 & 0 & 0 & 0 & 0 \\
\hline $80-84$ & 0 & 0 & 0 & 2 & 0 & 0 & 0 & 0 & 0 & 0 \\
\hline $85-89$ & 0 & 1 & 0 & 0 & 0 & 0 & 0 & 0 & 0 & 0 \\
\hline $90-94$ & 0 & 0 & 0 & 0 & 0 & 0 & 0 & 0 & 0 & 0 \\
\hline $95-99$ & 0 & 0 & 0 & 0 & 0 & 0 & 0 & 0 & 0 & 0 \\
\hline $100-$ & 0 & 4 & 0 & 6 & 0 & 0 & 0 & 0 & 0 & 0 \\
\hline & 2 & 124 & 10 & 65 & 42 & 23 & 34 & 27 & 19 & 10 \\
\hline
\end{tabular}

nAdj-THT, nAdj-RHT, Adj-THT, and Adj-RHT: (see Supp. Abbreviation)

Table 8a A CA-positive single tooth with dental caries in a single jaw (Using CHROMagar (a and b) )

\begin{tabular}{|c|c|c|c|c|c|}
\hline 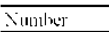 & C1 & 02 & C3pul & C3per & c 3res \\
\hline $1-4$ & 2 & 26 & 4 & 5 & 6 \\
\hline $5-9$ & 0 & 4 & 0 & 0 & 2 \\
\hline $10-14$ & 0 & 3 & 0 & 1 & 1 \\
\hline $15-19$ & 0 & 2 & 0 & 0 & 0 \\
\hline $20-24$ & 0 & 0 & 0 & 0 & 1 \\
\hline $25-29$ & 0 & 0 & 1 & 0 & 0 \\
\hline $30-34$ & 0 & 1 & 0 & 1 & 0 \\
\hline $35-39$ & 0 & 0 & 0 & 1 & 0 \\
\hline $40-44$ & 0 & 0 & 0 & 0 & 0 \\
\hline $45-49$ & 0 & 0 & 0 & 1 & 0 \\
\hline $50-54$ & 0 & 0 & 0 & 0 & 0 \\
\hline $55-59$ & 0 & 0 & 0 & 0 & 0 \\
\hline $60-64$ & 0 & 0 & 0 & 0 & 1 \\
\hline $65-69$ & 0 & 0 & 0 & 0 & 0 \\
\hline $70-74$ & 0 & 0 & 0 & 0 & 0 \\
\hline $75-79$ & 0 & 0 & 0 & 1 & 0 \\
\hline $80-84$ & 0 & 0 & 0 & 1 & 0 \\
\hline $85-89$ & 0 & 0 & 0 & 0 & 0 \\
\hline $90-94$ & 0 & 0 & 0 & 0 & 0 \\
\hline $95-99$ & 0 & 0 & 0 & 0 & 0 \\
\hline \multirow[t]{2}{*}{$100-$} & 0 & 0 & 0 & 1 & 0 \\
\hline & 2 & 36 & 5 & 12 & 11 \\
\hline
\end{tabular}

Table 8b A CA-positive single tooth with dental caries in the whole jaw

\begin{tabular}{cccccc}
\hline Nurulner & C1 & C2 & C3pul & C3per & C 3 rest \\
\hline $1-4$ & 2 & 17 & 4 & 4 & 4 \\
$5-9$ & 0 & 4 & 0 & 0 & 1 \\
$10-14$ & 0 & 3 & 0 & 1 & 1 \\
$15-19$ & 0 & 2 & 0 & 0 & 0 \\
$20-24$ & 0 & 0 & 0 & 0 & 1 \\
$25-29$ & 0 & 0 & 1 & 0 & 0 \\
$30-34$ & 0 & 1 & 0 & 0 & 0 \\
$35-39$ & 0 & 0 & 0 & 1 & 0 \\
$40-44$ & 0 & 0 & 0 & 0 & 0 \\
$45-49$ & 0 & 0 & 0 & 1 & 0 \\
$50-54$ & 0 & 0 & 0 & 0 & 0 \\
$55-59$ & 0 & 0 & 0 & 0 & 0 \\
$60-64$ & 0 & 0 & 0 & 0 & 0 \\
$65-69$ & 0 & 0 & 0 & 0 & 0 \\
$70-74$ & 0 & 0 & 0 & 0 & 0 \\
$75-79$ & 0 & 0 & 0 & 1 & 0 \\
$80-84$ & 0 & 0 & 0 & 0 & 0 \\
$85-89$ & 0 & 0 & 0 & 0 & 0 \\
$90-94$ & 0 & 0 & 0 & 0 & 0 \\
$95-99$ & 0 & 0 & 0 & 0 & 0 \\
$100-$ & 0 & 0 & 0 & 0 & 0 \\
\hline & 2 & 27 & 5 & 8 & 7
\end{tabular}


Table 9 The stage of dental caries and colony count immediately after the shift to positive from negative in the first examination (Using CHROMagar)

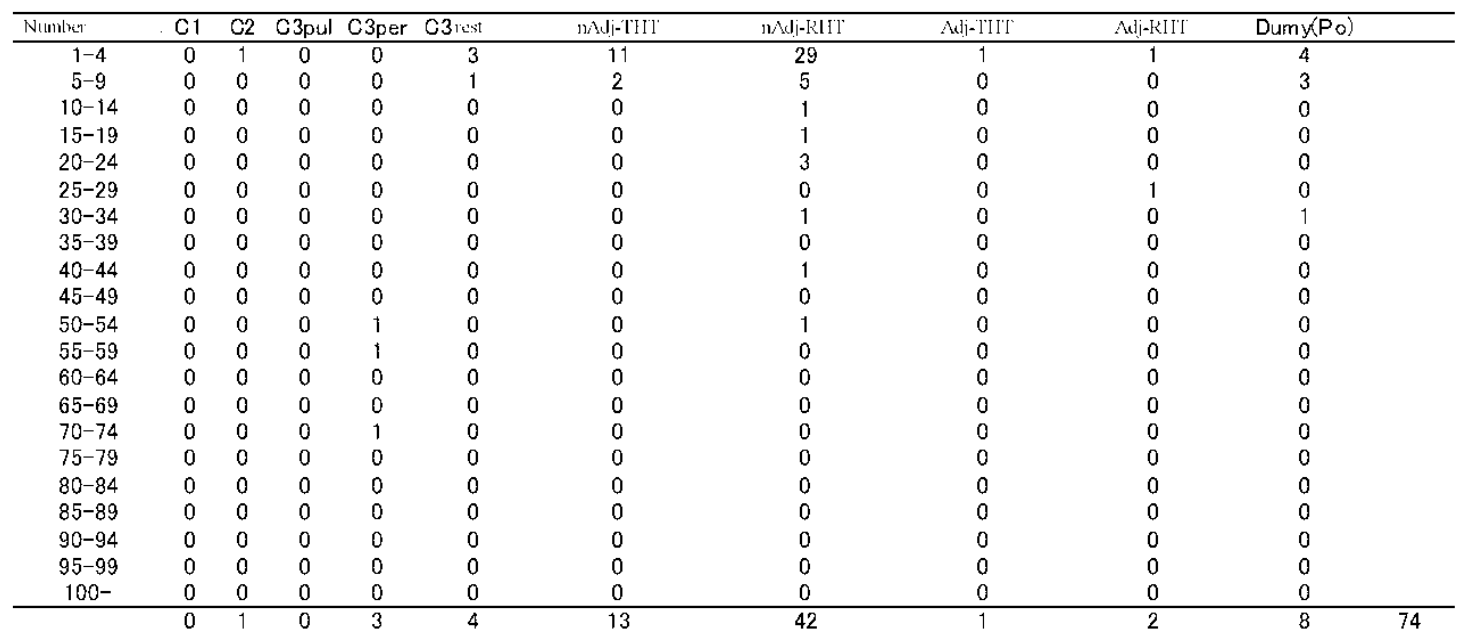

Table 10a CA-positive single healthy tooth in a single jaw (Using CHROMagar)

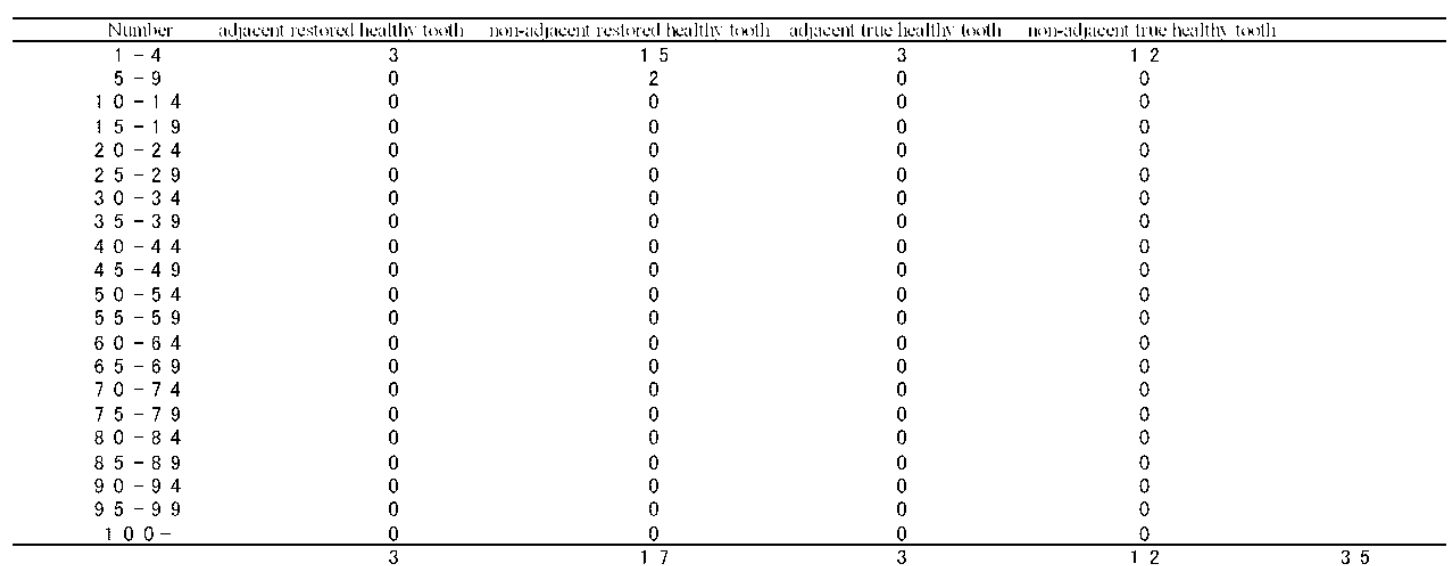

Table 10b CA-positive single healthy tooth in the whole jaw (Using CHROMagar)

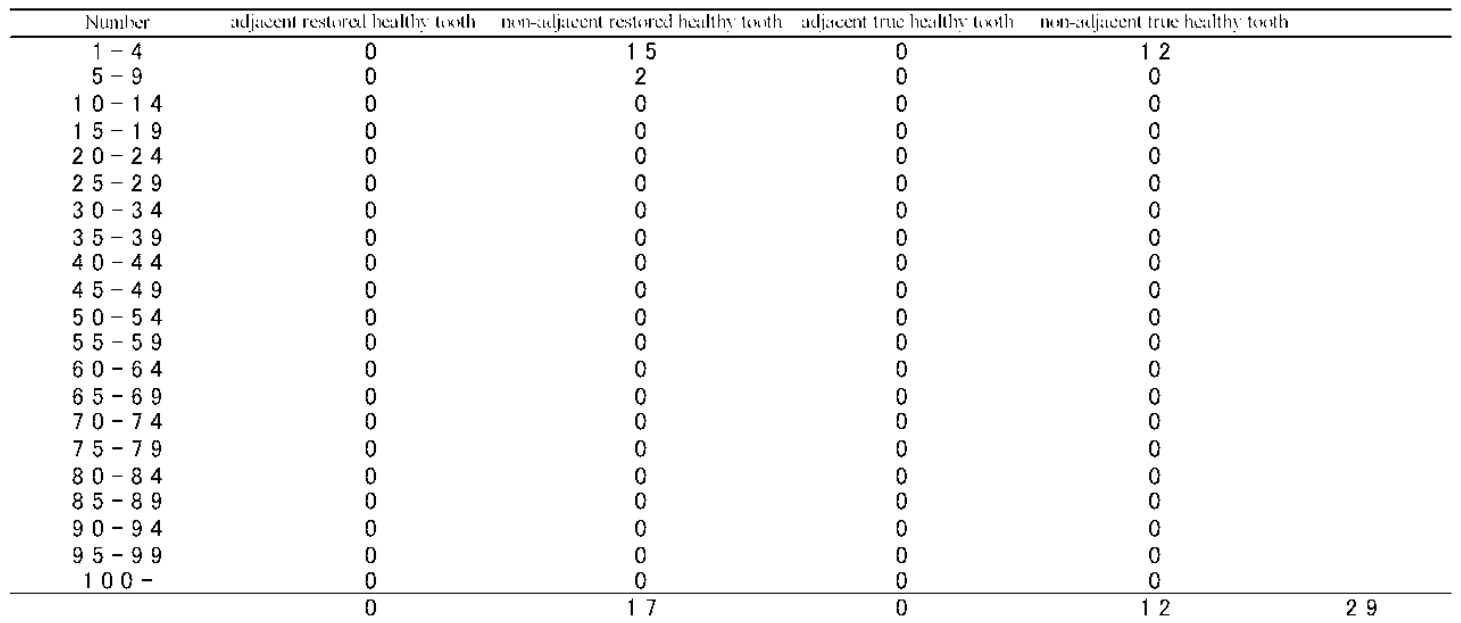




\section{Acknowledgements}

I would like to thank the staff at Nonomura Dental Clinic for their clinical assistance; Dr. Sachiko Nisida and Dr. Hiroko Kamide for their assistance with language editing; Miss Haruko Kobayashi, Miss Manami Niwa, and Mr. Keita Taki for searches for references, Mrs. Tomomi Okuda and Miss Saori Nishida for their assistance with the photographs and figures, and Mrs. Kaori Sato, Mrs. Tomomi Okuda, Miss Haruki Kuwayama, Dr. Tomohiro Yosida, and Dr. Haruna Isikawa for their assistance with editing text, figure, and statistical data.

\section{References}

1 Odds F (1988) Candida and Candidosis: a review and bibliography. Bailliere Tindale, London. 2 Bodey GP (1993) Candidiasis Pathogenesis, Diagnosis and Treatment $2^{\text {nd }}$ Ed. Raven Press, New York.

3 Kett, D., Dimopoulos, G., Azoulay, E. et al. Demographic and outcome differences in ICU patients with proven invasive candidiasis, possible invasive candidiasis and probable candida colonization: analysis of the EPIC II study population. Crit Care 15, P238 (2011). https://doi.org/10.1186/cc9658 4 Timsit JF, Schwebel C, Styfalova et. al (1999) Impact of bronchial colonization with Candida spp. on the risk of bacterial ventilator-associated pneumonia in the ICU: the FUNGIBACT prospective cohort study. Intensive Care Med 45:834-843. doi: 10.1007/s00134-019-05622-0

5 Ricard JD, Roux D (2012) Candida colonization in ventilated ICU patients: no longer a bystander! Intensive Care Med 38:1243-1245. https://doi.org/10.1007/s00134-012-2587-z

6 Hamet M, Pavo A, Dalle F et al (2012) Candida spp. airway colonization could promote antibiotic-resistant bacteria selection in patients with suspected ventilator-associated pneumonia. Intens Care Med 38: 1272-1279. doi: 10.1007/s00134-012-2584-2

7 Delisle MS, Williamson DR, Perreault MM et al (2008) The clinical significance of Candida colonization of respiratory tract secretions in critically ill patients. J Crit Care 23: 11-17. https://doi.org/10.1016/j.jcrc.2008.01.005

8 Tan X, Zhu S, Yan D et al (2016) Candida spp. airway colonization: A potential risk factor for Acinetobacter baumannii ventilator-associated pneumonia. Med Mycol 54: 557566. https://doi.org/10.1093/mmy/myw009

9 Elie A, François J, Arnaud ML et al (2006) Candida Colonization of the Respiratory Tract and Subsequent Pseudomonas Ventilator-Associated Pneumonia. Chest 129: 110-117.

10 Nseir S, Jozefowicz E, Cavestri B et al (2007) Impact of antifungal treatment on CandidaPseudomonas interaction: a preliminary retrospective case-control study. Intensive Care Med 33:137-142. DOI: 10.1007/s00134-006-0422-0

11 Haron E, Vartivarian S, Anaissie E et al (1993) Primary Candida pneumonia. Experience at a 
large cancer center and review of the literature. Medicine (Baltimore) 72:137-142.

12 Buehler PK, Zinkernagel AS, Hofmaenner DA et al (2020) Bacterial pulmonary superinfections are associated with unfavourable outcomes in critically ill COVID-19 patients. medRxiv https://www.medrxiv.org/content/10.1101/2020.09.10.20191882v1. doi: https://doi.org/10.1101/2020.09.10.20191882

13 Falsetta ML, Klein MI, Colonne PM et al (2014) Symbiotic relationship between Streptococcus mutans and Candida albicans synergizes virulence of plaque biofilms in vivo. Infect Immun 82: 1968-1981. DOI: 10.1128/IAI.00087-14

14 Y. Nonomura (2019) Measurement and self-operating computer of the leukocyte continuum as a fixed space-time continuum in inflammation. bioRxiv http://biorxiv.org/cgi/content/short/543611v2 15 Szeigoleit F, Szeigoleit TA, Wetzel WE (1999) Effect of dental treatment and/or local application of amphotericin B to carious teeth on oral colonization by Candida. Med Mycol 37: 345-350. doi: 10.1046/j.1365-280x.1999.00242.x.

16 Krasse B (1954) The relationship between Lactobacilli, Candida and Streptococci and dental caries. Examination of saliva and plaque material collected on the same occasion. Odontol Revy 5: 241-261.

17 Glass RL (1951) The occurrence of yeasts in the saliva of children. J Dent Res 30: 468.

18 Moalic E, Gestalin A, Quinio D et al (2001) The extent of oral fungal flora in 353 students and possible relationships with dental caries. Caries Res 35: 149-155. DOI: 10.1159/000047447

19 Fosdick LS, Hansen HL (1936) Theoretical considerations of carbohydrate degradation in relation to dental caries. J Am Dent Asso 401:23.

20 Knighton H (1939) A study of monilia and other yeastlike organisms found in the oral cavity. J Dent Res 18: 103 .

21 Lilienthal B (1970) Studies of the flora of the mouth. III. Yeast-like organisms: some observations on their incidence in the mouth. Aust J Exp Biol Med Sci 28: 279-286.

22 Arendorf TM, Walker DM (1980) The prevalence and intra-oral distribution of Candida albicans in man. Arch Oral Biol 25: 1-10. doi: 10.1016/0003-9969(80)90147-8

23 Food Sanitation Act of Japan. Article 6 paragraph 3.

https://www.mhlw.go.jp/web/t_doc?dataId=78330000\&dataType $=0 \&$ pageNo $=1$

24 Water Supply Act of Japan. Article 4 paragraph 1.

https://elaws.e-gov.go.jp/search/elawsSearch/elaws_search/1sg0500/detail?lawId=332 AC000000017 7

25 Rieg G, Fu Y, Ibrahim AS et al (1999) Unanticipated heterogeneity in growth rate and virulence among Candida albicans AAF1 null mutants. Infect Immun 67: 3193-3198. doi: 10.1128/IAI.67.7 


\section{Infection Chain}

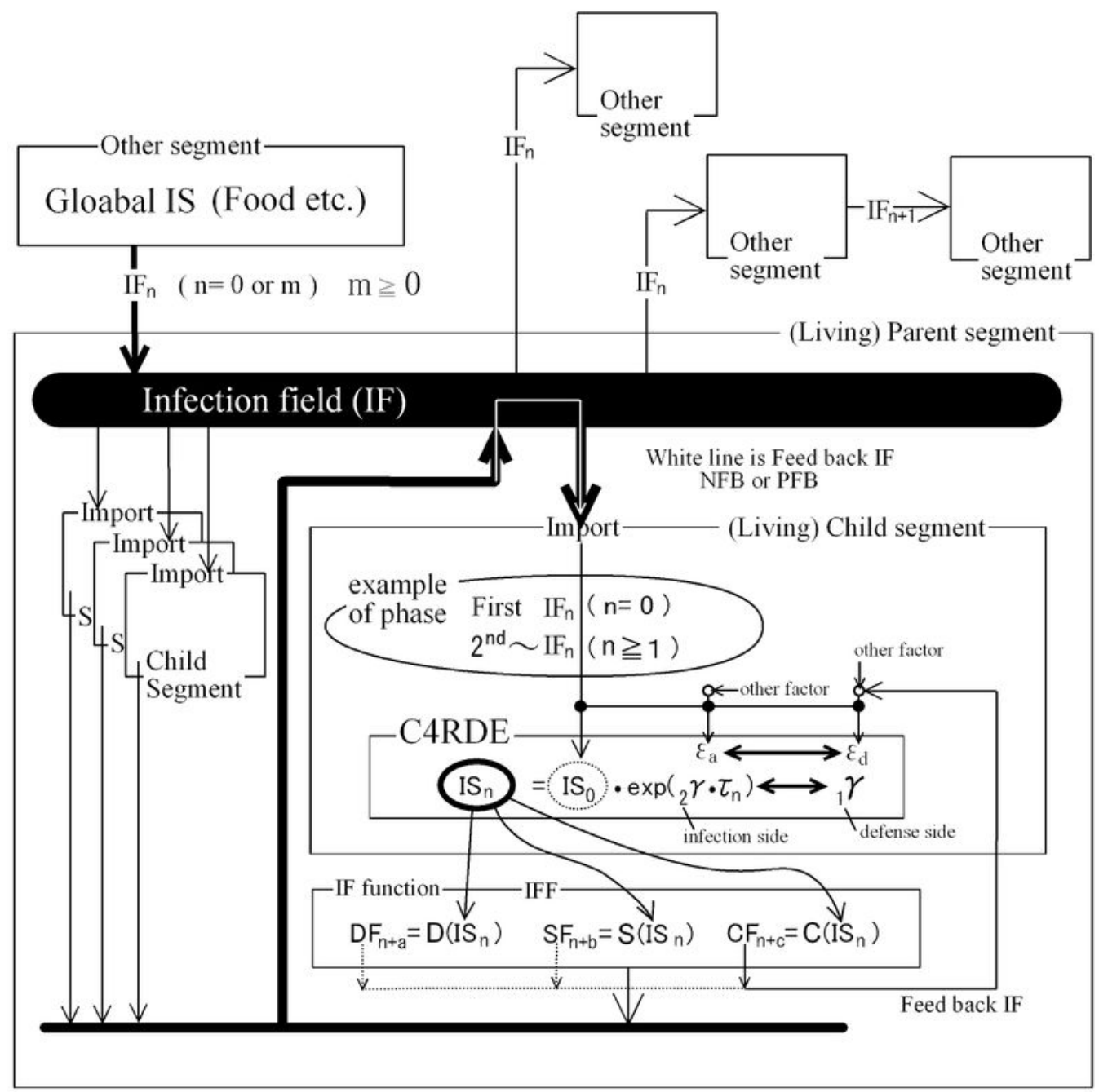

IF : Infection field

DF : Diffusion field

SF : Spread field

CF : Carrier field

IS : Infection source
RcF : Receptor field

RsF : Resistant field

SOI : Solution of infedction

SOD : Solution of defens

$\varepsilon_{\mathrm{a}}$ active coefficient $\mathrm{m}>0$ : integer

$\mathrm{n}>0$ : integer

$\mathrm{a}, \mathrm{b}, \mathrm{c}:$ integer

$\varepsilon_{\mathrm{d}}$ decline coefficient

\section{Figure 1}

Definition of infection chain (IC) and various segment(s) 


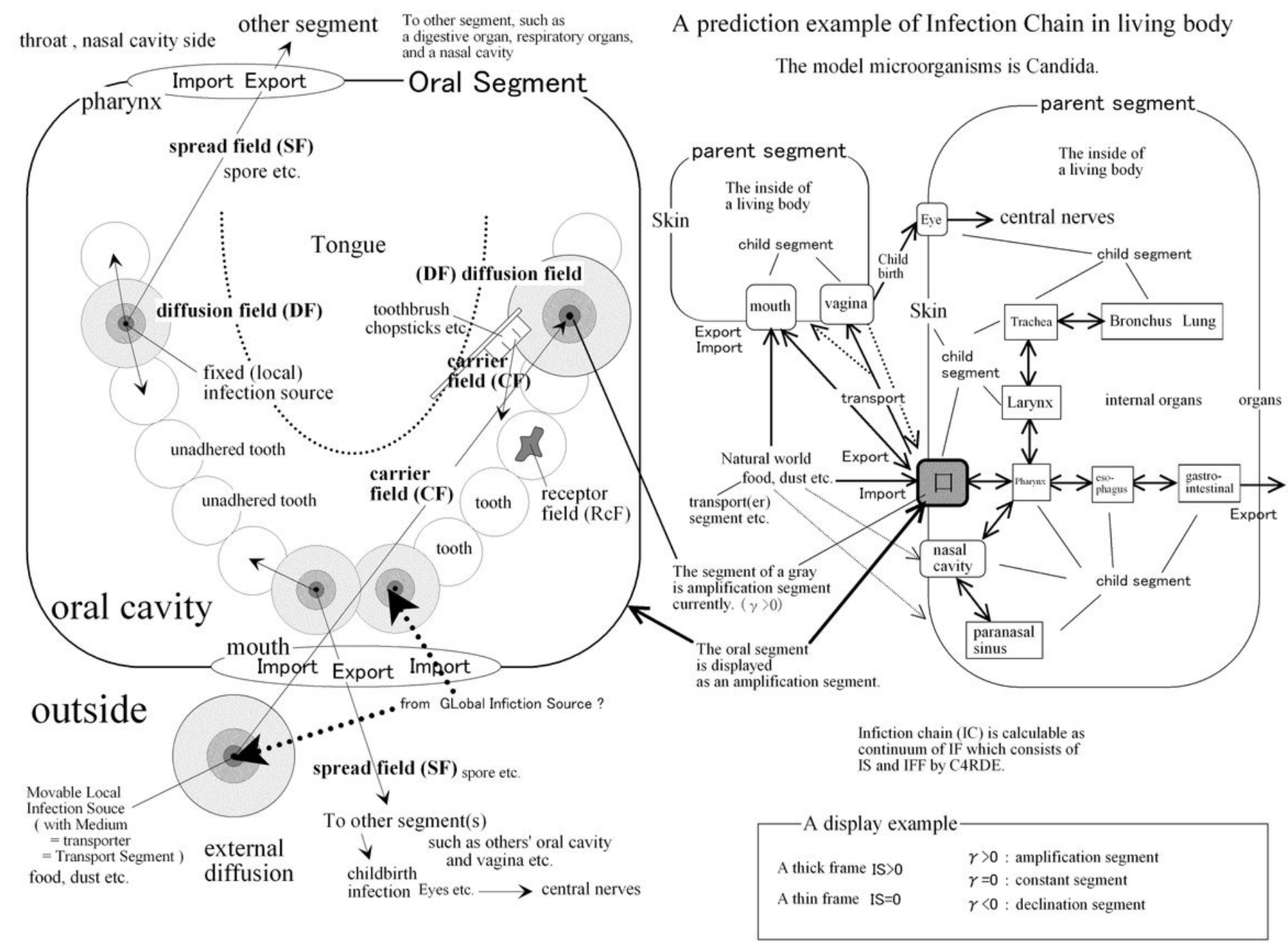

Figure 2

Each field in the oral segment and an example of the infection chain in living body. (In the case of Candida). 


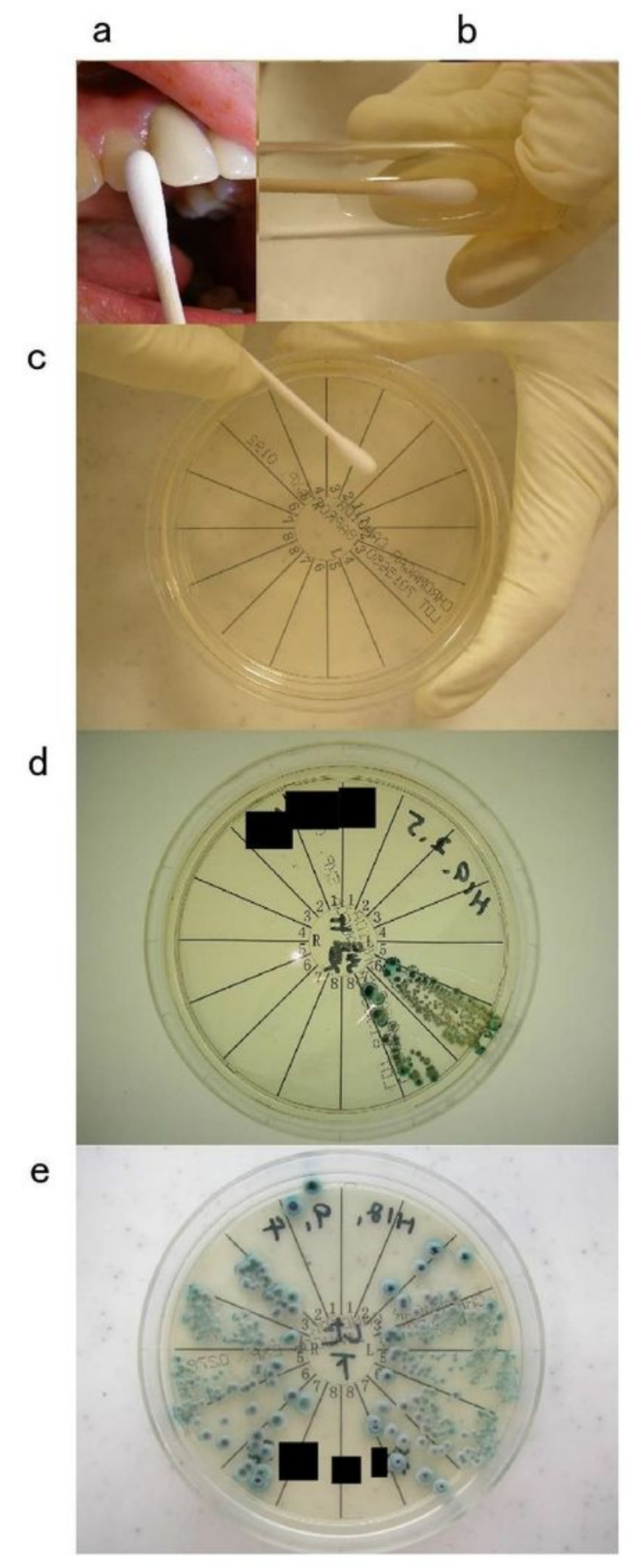

\section{Figure 3}

The sampling method of a sample by dental formula medium (DFM), a, A sterile swab of the teeth (crown). (Additionally, tracing the teeth cervix traces the entrance of the periodontal pocket, and samples soft tissues, such as the marginal gingiva and interdental papilla.) b, All teeth swabs were applied to medium (oral cavity unit examination). The all teeth examination (ATE), c, The sterile swab applied to the tooth that corresponded to each dental formula and the swab was applied to the area of dental formula 
on corresponding medium. The partial teeth examination (PTE) (tooth unit), $d$, As one or more CA-positive caries were observed, the infection source (IS) was tooth decay. e, As many CA-positive caries were observed, growth similar to that of endogenous microorganisms occurred. c-e, Dental formula medium (DFM) analyzes the sample according to the dental formula. The dental formula information on this DFM permitted space, time, and phase analyses (Table 3). The results in DFM clarified the infection by CA (Fig. $3 d$ and e).
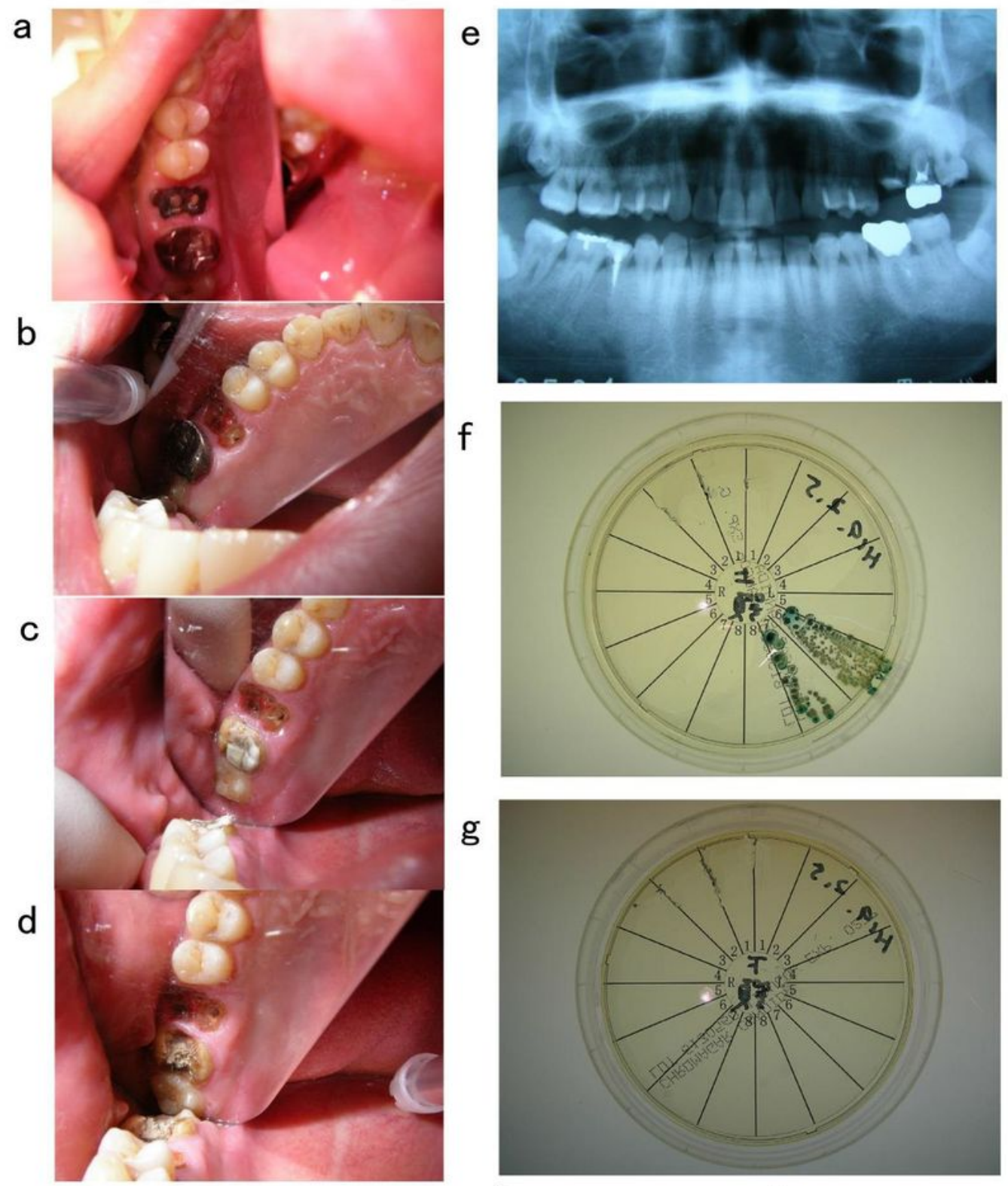

$\mathrm{f}$

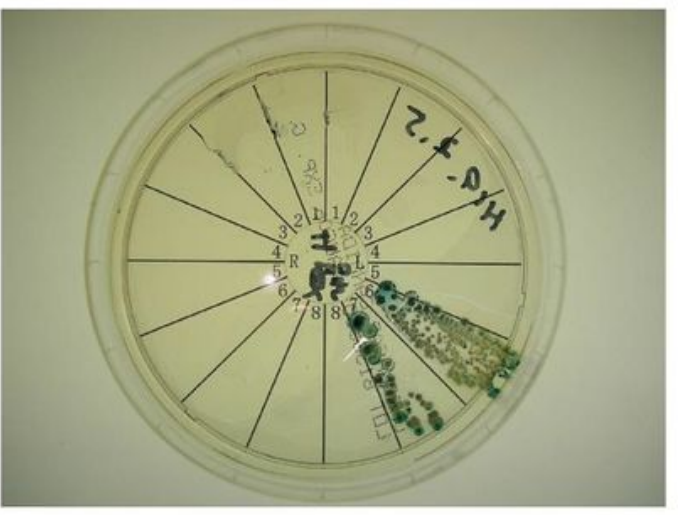

g
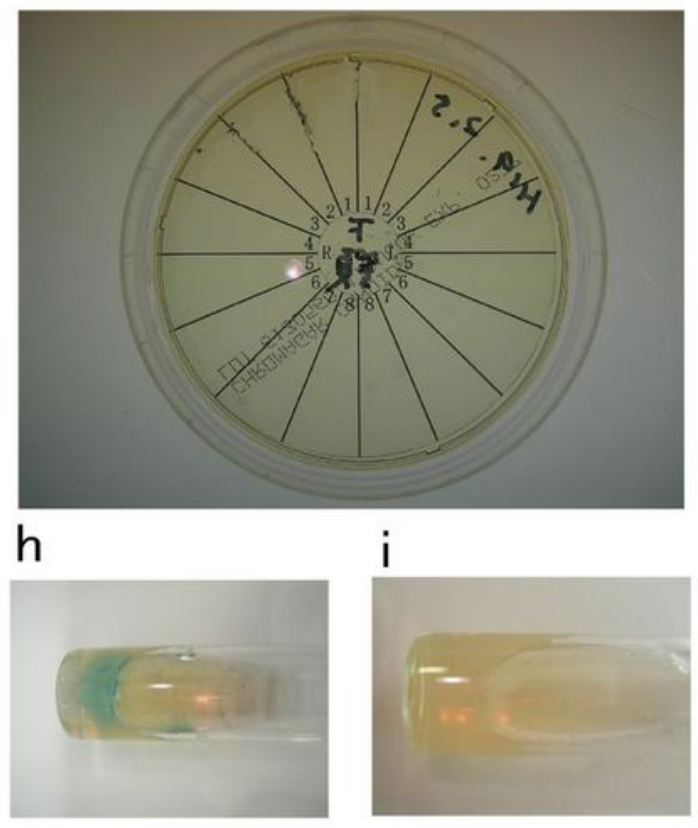
Disinfection case, a few positivity tooth, a, Before the operation, the upper left teeth No. 6 and 7 show dental-caries. X-ray findings showed caries in No 6 and 7. b, The caries in the upper left No. 6 tooth were removed. c, The upper left No. 7 crown was removed. $d$, The upper left No. 7 core was removed. e, Orthopantomography at the first dental examination. $f$, Dental formula medium upper jaw is CA colony level 4 (100- colony counts, the level of outbreak) in No. 6 tooth and level 3 (15-99 colony counts, fixing level with increased growth) in No. 7 tooth. g, Dental-formula medium lower jaw is clear. h, Before treatment, all teeth were CA-positive. i, After treatment, all teeth were CA-negative
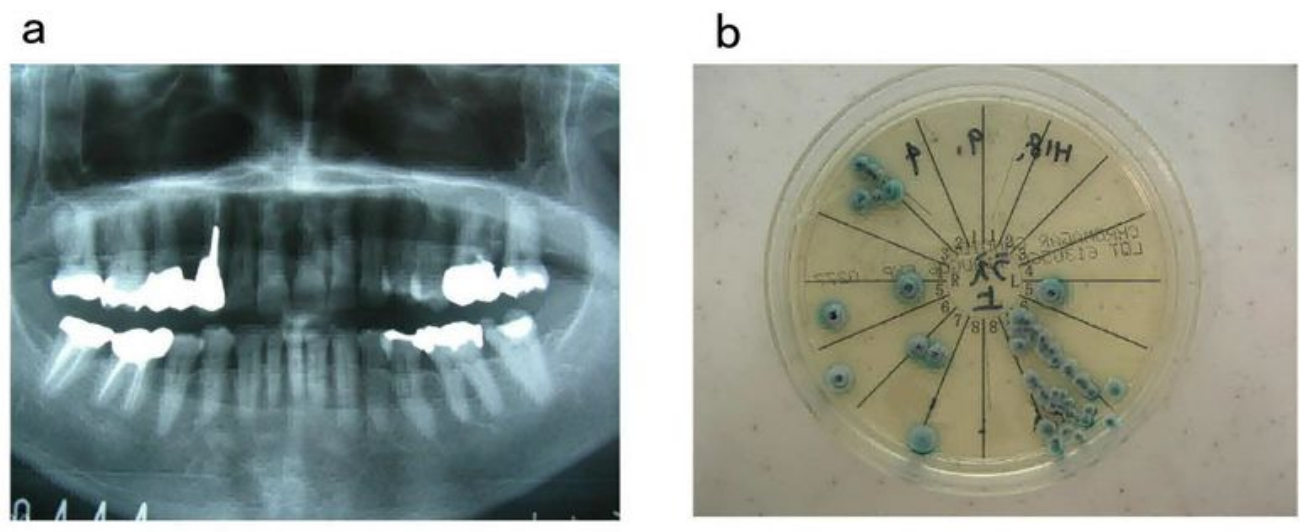

C
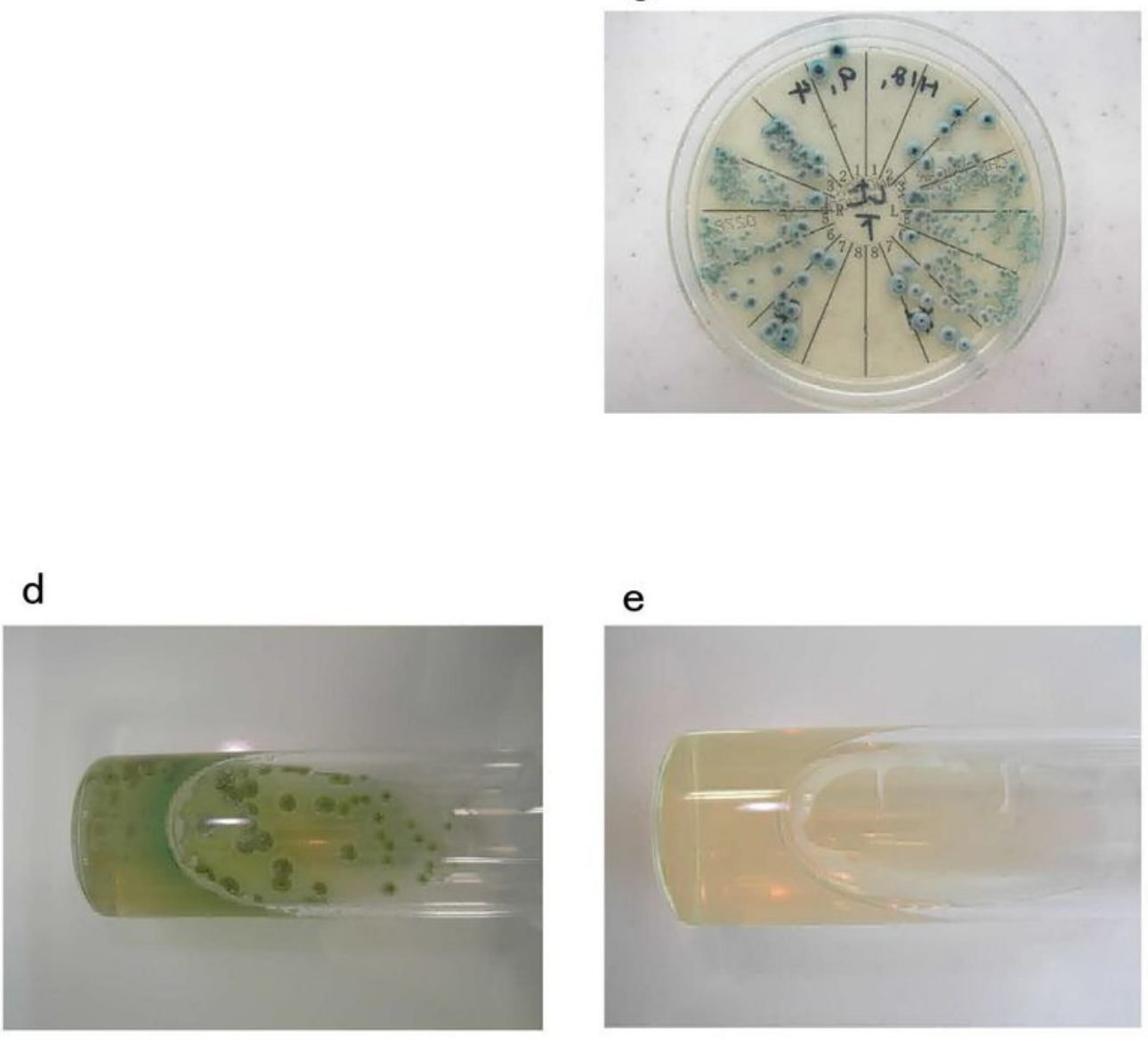


\section{Figure 5}

Disinfection case, many positive teeth, a, Orthopantomography at the first dental examination, $b$, Dentalformula medium upper jaw c, Dental-formula medium lower jaw. In this case, many teeth were CA infected. CA in the oral segment appears to be an endogenous microorganism. $d$, Before treatment, all teeth were CA-positive. e, After treatment, all teeth were CA-negative

\section{Supplementary Files}

This is a list of supplementary files associated with this preprint. Click to download.

- Supplementarylnformation1828E7.pdf 